\title{
Dynamical Coulomb Blockade as a Local Probe for Quantum Transport
}

\author{
Jacob Senkpiel, ${ }^{1}$ Jan C. Klöckner, ${ }^{2,3}$ Markus Etzkorn, ${ }^{1, \dagger}$ Simon Dambach, ${ }^{4}$ Björn Kubala, ${ }^{4, \ddagger}$ Wolfgang Belzig®,${ }^{3}$ \\ Alfredo Levy Yeyati $\odot,{ }^{5}$ Juan Carlos Cuevas $\odot,{ }^{5}$ Fabian Pauly $\odot,{ }^{2,3,8}$ Joachim Ankerhold, ${ }^{4}$ \\ Christian R. Ast $\oplus^{1, *}$ and Klaus Kern ${ }^{1,6}$ \\ ${ }^{1}$ Max-Planck-Institut für Festkörperforschung, Heisenbergstraße 1, 70569 Stuttgart, Germany \\ ${ }^{2}$ Okinawa Institute of Science and Technology Graduate University, Onna-son, Okinawa 904-0495, Japan \\ ${ }^{3}$ Fachbereich Physik, Universität Konstanz, 78457 Konstanz, Germany \\ ${ }^{4}$ Institut für Komplexe Quantensysteme and IQST, Universität Ulm, Albert-Einstein-Allee 11, 89069 Ulm, Germany \\ ${ }^{5}$ Departamento de Física Teórica de la Materia Condensada, Condensed Matter Physics Center (IFIMAC), and Instituto Nicolás \\ Cabrera, Universidad Autónoma de Madrid, 28049 Madrid, Spain \\ ${ }^{6}$ Institut de Physique, Ecole Polytechnique Fédérale de Lausanne, 1015 Lausanne, Switzerland
}

(Received 26 October 2019; accepted 24 March 2020; published 16 April 2020)

\begin{abstract}
Quantum fluctuations are imprinted with valuable information about transport processes. Experimental access to this information is possible, but challenging. We introduce the dynamical Coulomb blockade (DCB) as a local probe for fluctuations in a scanning tunneling microscope (STM) and show that it provides information about the conduction channels. In agreement with theoretical predictions, we find that the DCB disappears in a single-channel junction with increasing transmission following the Fano factor, analogous to what happens with shot noise. Furthermore we demonstrate local differences in the DCB expected from changes in the conduction channel configuration. Our experimental results are complemented by ab initio transport calculations that elucidate the microscopic nature of the conduction channels in our atomic-scale contacts. We conclude that probing the DCB by STM provides a technique complementary to shot noise measurements for locally resolving quantum transport characteristics.
\end{abstract}

DOI: 10.1103/PhysRevLett.124.156803

An important consequence of the downscaling of electronic circuits towards the atomic limit is the emergence of charge quantization effects [1-5]. The concomitant quantum fluctuations of charge and phase carry valuable information about transport processes [6], such as channel configuration, spin polarization, or effective charge [7-17]. Accessing them experimentally, however, for instance through shot-noise measurements [18] is quite challenging, but feasible [19-26]. Alternatively, the dynamical Coulomb blockade (DCB) is also a consequence of quantum fluctuations. It arises from the inelastic interaction of tunneling electrons with the local electromagnetic environment [2732], in which the junction is embedded [see Fig. 1(a)]. It appears when the thermal energy $k_{B} T$, with the temperature $T$ and the Boltzmann constant $k_{B}$, is on the order of or smaller than the charging energy $E_{C}=e^{2} / 2 C_{J}$ associated with the capacitance $C_{J}$ of the tunnel junction ( $e$ is the elementary charge). The DCB is directly observable in differential conductance data, where it manifests itself as a

Published by the American Physical Society under the terms of the Creative Commons Attribution 4.0 International license. Further distribution of this work must maintain attribution to the author(s) and the published article's title, journal citation, and DOI. Open access publication funded by the Max Planck Society.
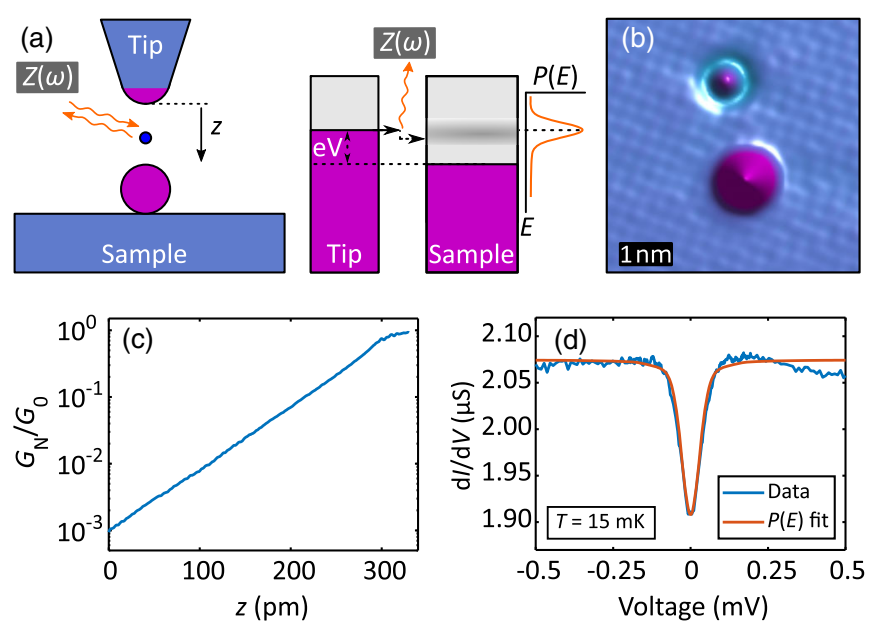

FIG. 1. (a) Schematic representation of an atomic tunnel junction in the DCB regime and corresponding energy diagram highlighting the environmental interaction. (b) Topography of a single $\mathrm{Al}$ adatom adsorbed on the $\mathrm{Al}(100)$ surface. The $\mathrm{Al}$ adatom is located in the lower half, in the upper part an intrinsic defect is visible. (c) Approach curve on the Al adatom with an Al tip (both in the normal conducting state) at a bias voltage well above the DCB dip. In (d) the dip in the normal conducting $d I / d V$ curve, prototypical for the $\mathrm{DCB}$, is shown with a $P(E)$ fit in the lowconductance limit. 
dip in the voltage range on the order of $E_{C} / e$ around zero bias [33-36], as, for example, at very low temperatures $(\lesssim 1 \mathrm{~K})$ in small capacitance (few fF) mesoscopic circuits [29-38].

In this Letter, we exploit the DCB in ultralow temperature scanning tunneling spectroscopy (STS) as a tool to locally identify the quantum transport characteristics of atomic-scale junctions all the way from the tunnel to the contact regime. First, we use a junction formed between two single atoms featuring a single dominant transport channel [39]. The DCB is seen at low transmission, but disappears with increasing transmission following the Fano factor of a single-channel junction [40]. Extending the measurements to a junction between a single atom on one side and two atoms on the other side, we find a different signature in the DCB dip. This indicates a direct influence of the set of nonvanishing channel transmissions $\tau_{i}$, also referred to as the mesoscopic PIN code characteristic for the junction [41], on the DCB. We conclude that DCB measurements in STS below $1 \mathrm{~K}$ provide direct access to the mesoscopic PIN code as a technique complementary to shot noise measurements $[14,40,42-45]$ as well as other techniques [46,47].

We first use the atomic manipulation capabilities of the scanning tunneling microscope (STM) to construct a junction between two single aluminum atoms [see Fig. 1(a)]. One $\mathrm{Al}$ atom is placed at the $\mathrm{Al}$ tip apex and one on the (100) surface of an Al crystal, as shown in the lower half of Fig. 1(b). By applying a magnetic field of $20 \mathrm{mT}$, the superconductivity in $\mathrm{Al}$ is quenched and we obtain a normal conducting junction at an experimental temperature $T$ of $15 \mathrm{mK}$ [48]. We can reproducibly and continuously tune the junction conductance up to the quantum of conductance $G_{0}=2 e^{2} / h$ (with Planck's constant $h$ ) by changing the tip-sample distance, as we illustrate in Fig. 1(c).

We start by studying the differential conductance $G(V)$ in the tunnel regime at bias voltage $V$, where the set point conductance $G_{N}=G_{0} \sum_{i} \tau_{i}=G_{0} \tau_{t}$ and $G_{N} \ll G_{0}$. As we show in Fig. 1(d) for $G_{N}=0.027 G_{0}$, the conductance exhibits a dip at low bias voltage, which is the typical signature of DCB. To verify this observation we analyze our data using the $P(E)$ theory $[29,37,49]$. In the $P(E)$ model, the interaction of tunneling charged particles with the environment is taken into account by the environmental impedance $Z(\omega)$, as shown schematically in Fig. 1(a). The obtained fit is indicated in Fig. 1(d) as an orange line. We find for the junction capacitance $C_{J}=21.7 \mathrm{fF}$ and for the effective temperature $T_{\text {eff }}=84.9 \mathrm{mK}$. The fit confirms that we operate in a low-impedance regime, where the zero frequency part of the environmental impedance is $R_{\text {env }}=$ $377 \Omega$ and much smaller than $1 / G_{0}=R_{Q}$ [40], resulting in a small reduction in conductance $\delta G(0)=G(0)-G_{N}$ at zero bias voltage of $\delta G(0) / G_{N}=-9 \%$. The modeling is detailed in the Supplemental Material [50].
This establishes the DCB in the tunneling regime at low conductances. However, as we approach the tip to the adatom on the sample, the conductance increases, and we observe a clear reduction in the DCB. The experimental data is shown in Fig. 2(a) for different conductance values ranging from $0.03 G_{0}$ close to $1 G_{0}$. The spectra have been normalized to the set point conductance $G_{N}$ in the voltage range outside of the DCB dip. The reduction in conductance at zero bias voltage $\delta G(0)$ gradually decreases until it disappears at the highest conductance. This suppression of the DCB as the channel transmission approaches the ballistic limit of perfect transmission $\left(\tau_{1} \rightarrow 1\right)$ has been observed in other types of quantum point contacts [33$35,58]$. It can be understood by considering the suppression of fluctuations in the number of electrons transmitted through the junction with increasing transmission, which is captured in the Fano factor $F=\sum_{i} \tau_{i}\left(1-\tau_{i}\right) / \sum_{i} \tau_{i}$. The relative change in conductance $\delta G(V) / G_{N}$ for weak coupling to the environment $Z(\omega)$ and at zero temperature was derived for a single-channel system in Ref. [40] and for multiple channels in Ref. [59]:

$$
\frac{\delta G(V)}{G_{N}}=-F \int_{e V}^{\infty} \frac{d \omega}{\omega} \frac{\operatorname{Re} Z(\omega)}{R_{Q}}
$$
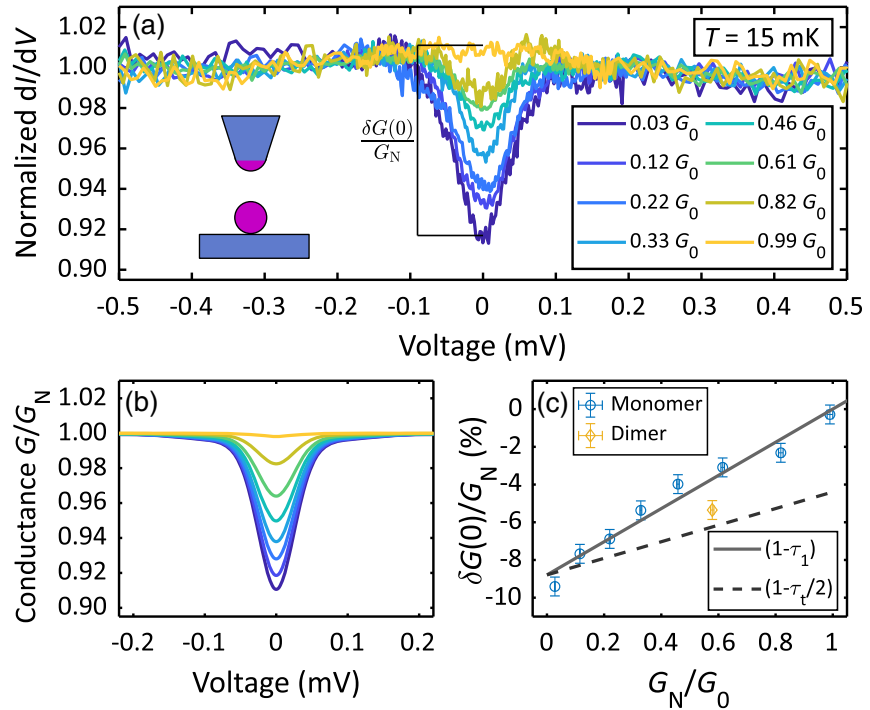

FIG. 2. DCB dip as a function of junction conductance. In (a) we present $d I / d V$ data with junction conductances ranging from $0.03 G_{0}$ up to $0.99 G_{0}$. The data is normalized to the conductance values outside of the DCB dip. (b) The theoretical dependence based on Ref. [40]. Parameters were determined by the $P(E)$ fit in Fig. 1(d), the color code corresponds to (a). (c) The $d I / d V$ reduction at zero bias $\delta G(0) / G_{N}$ dependent on junction conductance, is plotted as blue circles for the single atom and as a yellow diamond for the dimer. We added a linear fit to the data assuming a single-channel junction $\tau_{t}=\tau_{1}$, where the dip reduces in magnitude with increasing conductance as $\left(1-\tau_{1}\right)$ from its value in the tunneling limit. For comparison, a dashed line (1$\left.\tau_{t} / 2\right)$ is shown, representing the behavior of a corresponding junction with two equal channels $\tau_{1}=\tau_{2}=\tau_{t} / 2$. 
The integral in Eq. (1) shows that for a generally small environmental impedance $\operatorname{Re} Z(\omega) \ll R_{Q}$, as realized in the STM, the change in conductance will be comparatively small. In Fig. 2(b) we model the transmission-dependent DCB dip based on the theory in Ref. [40] for one transmission channel $\tau_{1}$ (see also Supplemental Material [50]). We use the same parameters for the environmental interaction as before in the $P(E)$ fit depicted in Fig. 1(d) and find good agreement with the data. The decrease of the experimental DCB dip with increasing conductance $G_{N}$ is shown in Fig. 2(c) as blue circles. Here, we plot the reduction $\delta G(0) / G_{N}$, which can be directly extracted from the experimental data [cf. Fig. 2(a)] and is independent of the $P(E)$ fit. It follows a $\left(1-\tau_{1}\right)$ dependence as expected from the Fano factor $F$ [cf. Eq. (1)] and verified through the linear fit. This finding of pronounced single-channel characteristics in a junction between two $\mathrm{Al}$ atoms is consistent with previous experimental results obtained using the subgap structure of the current in the superconducting state [39].

In order to understand the observation of a single channel and to elucidate its origin, we have performed quantum transport calculations within the Landauer-Büttiker approach for coherent transport using a method that combines density functional theory (DFT) with nonequilibrium Green's function (NEGF) techniques. In particular, this approach makes it possible to optimize the junction geometries, to compute their electronic structure and transport characteristics, including the transmission eigenchannels [60]. As in the experiment the $\mathrm{Al}$ sample is modeled as a (100) surface with an additional $\mathrm{Al}$ adatom. The structure of the tip oriented along a (100) direction, and the sample are displayed in Fig. 3(a). The channel transmissions $\tau_{i}$ were extracted as a function of tip-sample distance, as is visible in Fig. 3(b). We can clearly see that the calculations reproduce the single-channel nature of the atomic $\mathrm{Al}$ contact. The transmissions of the second and third channel $\tau_{2}$ and $\tau_{3}$ are about 2 orders of magnitude smaller than those of the dominant channel $\tau_{1}$ over the full range of $z$ values considered, in contrast to the situation in break junction experiments [61-63]. Since higher order channels contribute even less, we focus on $\tau_{1}, \tau_{2}, \tau_{3}$ in the following [64], corresponding to the valence states of $\mathrm{Al}[63,65]$. Further insight can be obtained by calculating the complex-valued scattering-state wave functions of the transmission channels [66,67], as shown in Fig. 3(c). For an electron wave impinging on the contact from the substrate, we observe that the dominant first transport channel is of $\sigma$ symmetry in the narrowest part of the junction. In comparison, the second and third channels exhibit a $\pi$ shape when viewed along the transport direction. Thus, the theoretically calculated PIN code is $(0.575,0.003,0.001)$, which implies that the first channel provides $99.3 \%$ of the total transmission. Similar theoretical results were obtained for a junction geometry with an atomically sharp tip oriented along the (111) direction (see Supplemental Material [50]). From the
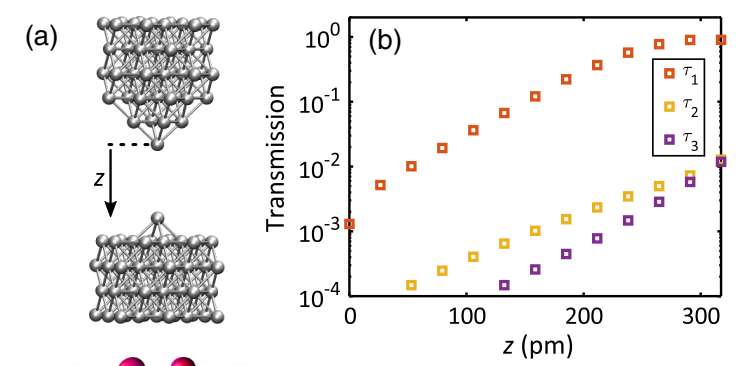

(c)
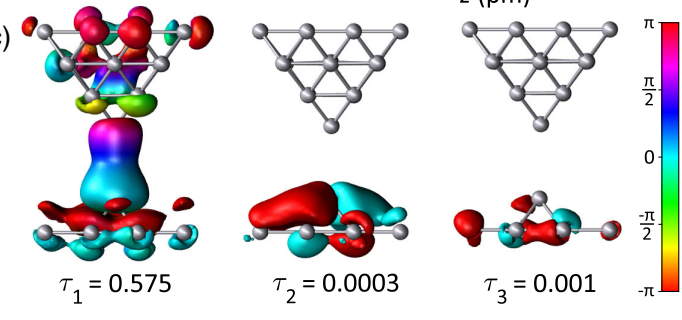

FIG. 3. Simulation of a single-atom Al junction based on the DFT-NEGF approach. In (a) the assumed geometry is displayed. Tip and surface are oriented in the (100) direction. (b) The obtained approach curve. The total transmission $\tau_{t}$ is determined to excellent approximation by the transmission of the first channel $\tau_{1}$. Transmissions of channels two $\tau_{2}$ and three $\tau_{3}$ are about 2 orders of magnitude reduced in comparison to those of channel one. In (c) the calculated wave functions of channels 1, 2, and 3 are shown, impinging on the junction from the sample at $0.58 G_{0}$. Colors encode the phase of the complex-valued channel wave functions, while identical absolute values are visualized through the isosurface.

experimental data at higher transmission, we estimate that channels beyond the first contribute no more than 3\% to the total transmission at $0.99 G_{0}$, which agrees nicely with the theoretical results.

Exploiting the local atomic resolution and manipulation capabilities of the STM, we can build more complicated atomic structures on the surface such as a dimer of $\mathrm{Al}$ atoms. This is visualized in Fig. 4(a), where the dimer is marked in purple. We placed two $\mathrm{Al}$ atoms on face-centered cubic lattice sites parallel to the atomic rows, separated by one site. Approaching the tip over the bridge position of the dimer, we anticipate more than one significant transport channel in the junction. The DCB spectrum for the dimer is shown in Fig. 4(b) as a blue line together with a measurement on a monomer. Both of them are taken at a total conductance of $0.58 G_{0}$. The characteristic dip at zero bias voltage is clearly visible. Comparing the $d I / d V$ curve on the dimer with the one on the monomer, we find that the DCB dip for the dimer is much more pronounced. From the experimental data on the dimer we extract a conductance reduction at zero voltage of $\delta G(0) / G_{N}=-5.4 \%$, whereas the reduction on the monomer at the same $G_{N}$ value is $\delta G(0) / G_{N}=-3.7 \%$ [68]. Considering the identical total conductance, this is only possible if the number of transmissive channels has changed, such that the first channel has a lower transmission, which leads to a more pronounced DCB dip. Analyzing the dimer DCB dip, we 


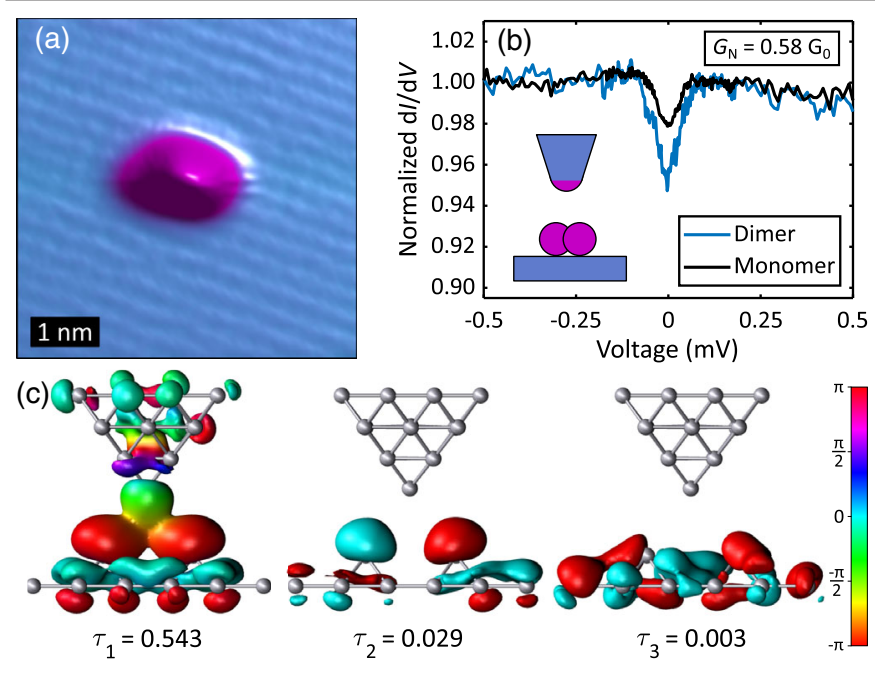

FIG. 4. Transport through a dimer on the substrate surface. (a) Topography of the dimer. (b) Comparison of experimentally determined transport through a monomer on the substrate surface to transport through a dimer. The DCB dip on the dimer is significantly stronger than those on the monomer at a similar total conductance of $0.58 G_{0}$. (c) Calculated wave functions and channel transmissions for the surface dimer for electrons entering from the substrate, at a total transmission corresponding to the experimental conductance in (b). Tip and surface are oriented along the (100) direction. The representation of the transmission channel wave functions is identical to Fig. 3(c).

consider two contributing channels and experimentally find a PIN code of $(0.46,0.12)$, with an estimated uncertainty of \pm 0.05 for each channel.

Like for the monomer, we simulated the junction with the dimer to gain further insight into the microscopic origin of the transport channel configuration. The wave functions for the channels 1, 2 and 3 are displayed in Fig. 4(c) for $G_{N}=0.58 G_{0}$. The simulations yield a PIN code of $(0.543$, $0.029,0.003)$ for a $(100)$-oriented tip and $(0.540,0.034$, $0.004)$ for a (111)-oriented tip, in acceptable agreement with the experimental findings (see details below). For these configurations, $93.6 \%$ and $93.1 \%$ of the total transmission is carried by the first channel, respectively. This is in contrast to the simulations of the monomer at the same conductance [(100)-tip orientation: $(0.575,0.003,0.001)$; (111)-tip orientation: $(0.576,0.002,0.002)]$, where in both configurations channel 1 contributes more than $99 \%$ to the total transmission (see Supplemental Material [50]). Hence, the transport channel configuration has clearly changed between the monomer and the dimer. Even if our calculations predict that the transport between the dimer and tip is dominated by the first channel, the transmission of the second channel is enhanced by one order of magnitude with respect to the monomer. For this reason we regard the dimer-tip system as a two-channel junction.

The experimentally observed more pronounced DCB dip on the dimer than on the monomer is in agreement with these predictions. Quantitative differences between theory and experiment for the dimer may arise from uncertainties in the precise atomic configuration of the tip, e.g., deviations from a perfect single-atom apex. Such deviations are visible as a small distortion of the dimer in Fig. 4(a). Considering that a change of the tip in the calculations, which is expected to be hardly visible in the topography, already yields a 14\% change of $\tau_{2}$ demonstrates the sensitivity of our method.

To test the range of applicability of this technique, we measured the DCB also in the high-temperature limit. This data was taken on the crystal surface at $1.32 \mathrm{~K}$ and $0.13 G_{0}$, see Fig. 5(a). We model it with the same values of the parameters describing the electromagnetic environment in the $P(E)$ fit of the DCB in Fig. 1(d), only changing the temperature. While we find overall consistency between low- and high-temperature data and modeling, the dip at high temperature only reduces the conductance by about $1 \%$, making it more challenging to detect changes. To reduce the error bar on these measurements, the strength of the DCB needs to be significantly increased. This can be achieved by changing the junction capacitance, since a smaller $C_{J}$ yields a more pronounced dip. To illustrate the effect, we model the DCB within an experimentally relevant range of $C_{J}$ between 1 and $60 \mathrm{fF}$ and temperatures between $10 \mathrm{mK}$ and $1.5 \mathrm{~K}$ based on the $P(E)$ model [36]. All other parameters are kept at the values used above. The obtained dependence is representative for the tunneling regime $\left(\tau_{t} \ll 1\right)$ and is plotted in Fig. 5(b). Our calculation shows that even in the high-temperature limit, smallcapacitance junctions should yield a reasonable $\delta G(0) / G_{N}$. The junction capacitance can be changed by adjusting the macroscopic tip geometry [36]. Therefore, we surmise that a number of experiments would profit by probing local PIN code variations using the DCB. The trade-off in energy resolution due to the reduced capacitance is likely not an issue at higher temperatures (of around $1 \mathrm{~K}$ ) due to prevalent thermal broadening [36]. In this sense using the DCB to extract the transport
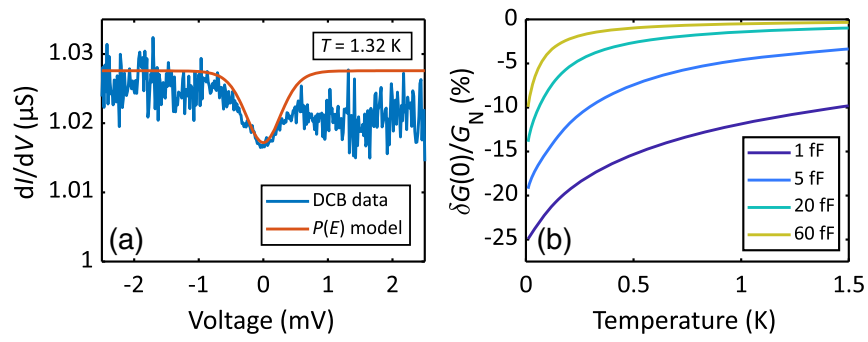

FIG. 5. Temperature range, where the DCB effect is detectable in the STM. (a) Measurement of the DCB dip at about $1.32 \mathrm{~K}$, where it is still accessible with our junction capacitance of $21.7 \mathrm{fF}$. The orange line models the data based on the $P(E)$ function, using the same values as for the low-temperature data, only adjusting $T$. (b) Calculated values of $\delta G(0) / G_{N}$ for a range of $C_{J}$ and $T(10 \mathrm{mK}$ to $1.5 \mathrm{~K})$ predict that clear changes of the DCB should be observable even above $1 \mathrm{~K}$. 
characteristics becomes a viable, complementary alternative to shot-noise measurements.

In summary, we have shown an alternative path to access transport properties on the atomic scale based on the DCB, applicable with standard measurement electronics. Apart from the Fano factor dependence of the DCB in the STM, we have demonstrated that it can be used in normalconducting junctions to extract local changes of the mesoscopic PIN code, where Andreev reflections cannot be exploited $[62,63]$. As a perspective, the DCB measurements in the STM should be further extendable to other properties accessible by shot noise, including the spin polarization of tunneling particles and possibly also the determination of their effective charge.

We gratefully acknowledge stimulating discussions with Elke Scheer and Alexander Weismann. This work was funded in part by the ERC Consolidator Grant AbsoluteSpin (Grant No. 681164). J. C. K., W. B., and F. P. thank the Collaborative Research Center (SFB) 767 of the Deutsche Forschungsgemeinschaft (DFG) for financial support. Part of the numerical modeling was performed using the computational resources of the bwHPC program, namely, the bwUniCluster and the JUSTUS HPC facility. A. L. Y. and J. C. C. acknowledge funding from the Spanish MINECO (Grants No. FIS2017-84057-P and No. FIS201784860-R) and from the "María de Maeztu" Programme for Units of Excellence in R\&D (MDM-2014-0377). J. A., S. D., and B.K. acknowledge financial support from the Zeiss-Foundation, the Institute for Quantum Science and Technology (IQST), and the Deutsche Forschungsgemeinschaft (DFG) under AN336/11-1.

* Corresponding author.

c.ast@fkf.mpg.de

${ }^{\dagger}$ Present address: Institut für Angewandte Physik, TU Braunschweig, 38106 Braunschweig, Germany.

*Present address: Institute of Quantum Technologies, German Aerospace Center (DLR), 89069 Ulm, Germany.

${ }^{\S}$ Present address: Institute of Physics, University of Augsburg, 86135 Augsburg, Germany.

[1] B. J. van Wees, H. van Houten, C. W. J. Beenakker, J. G. Williamson, L. P. Kouwenhoven, D. van der Marel, and C. T. Foxon, Quantized Conductance of Point Contacts in a Two-Dimensional Electron Gas, Phys. Rev. Lett. 60, 848 (1988).

[2] D. A. Wharam, T. J. Thornton, R. Newbury, M. Pepper, H. Ahmed, J. E. F. Frost, D. G. Hasko, D. C. Peacock, D. A. Ritchie, and G. A. C. Jones, One-dimensional transport and the quantisation of the ballistic resistance, J. Phys. C 21, L209 (1988).

[3] R. Landauer, Conductance determined by transmission: Probes and quantised constriction resistance, J. Phys. Condens. Matter 1, 8099 (1989).

[4] R. W. Keyes, Physical limits of silicon transistors and circuits, Rep. Prog. Phys. 68, 2701 (2005).
[5] W. Nawrocki, Physical limits for scaling of electronic devices in integrated circuits, in Physical Properties of Nanosystems, edited by J. Bonca and S. Kruchinin (Springer Netherlands, Dordrecht, 2011), p. 79.

[6] Y. V. Nazarov, Quantum Noise in Mesoscopic Physics, Nato Science Series II: Mathematics, Physics and Chemistry Vol. 97 (Springer Netherlands, Dordrecht, 2002).

[7] L. Saminadayar, D. C. Glattli, Y. Jin, and B. Etienne, Observation of the $e / 3$ Fractionally Charged Laughlin Quasiparticle, Phys. Rev. Lett. 79, 2526 (1997).

[8] R. de-Picciotto, M. Reznikov, M. Heiblum, V. Umansky, G. Bunin, and D. Mahalu, Direct observation of a fractional charge, Natue (London) 389, 162 (1997).

[9] J. M. van Ruitenbeek, Shot noise and channel composition of atomic-sized contacts, in Quantum Noise in Mesoscopic Physics, edited by Y. V. Nazarov (Springer Netherlands, Dordrecht, 2003), p. 33.

[10] M. Kumar, O. Tal, R. H. M. Smit, A. Smogunov, E. Tosatti, and J. M. van Ruitenbeek, Shot noise and magnetism of Pt atomic chains: Accumulation of points at the boundary, Phys. Rev. B 88, 245431 (2013).

[11] R. Vardimon, M. Klionsky, and O. Tal, Experimental determination of conduction channels in atomic-scale conductors based on shot noise measurements, Phys. Rev. B 88, 161404(R) (2013).

[12] M. Hashisaka, T. Ota, K. Muraki, and T. Fujisawa, ShotNoise Evidence of Fractional Quasiparticle Creation in a Local Fractional Quantum Hall State, Phys. Rev. Lett. 114, 056802 (2015).

[13] R. Vardimon, M. Klionsky, and O. Tal, Indication of complete spin filtering in atomic-scale nickel oxide, Nano Lett. 15, 3894 (2015).

[14] A. Burtzlaff, A. Weismann, M. Brandbyge, and R. Berndt, Shot Noise as a Probe of Spin-Polarized Transport Through Single Atoms, Phys. Rev. Lett. 114, 016602 (2015).

[15] R. Vardimon, M. Matt, P. Nielaba, J. C. Cuevas, and O. Tal, Orbital origin of the electrical conduction in ferromagnetic atomic-size contacts: Insights from shot noise measurements and theoretical simulations, Phys. Rev. B 93, 085439 (2016).

[16] S. Pradhan and J. Fransson, Shot noise as a probe of spincorrelated transport through single atoms, Phys. Rev. B 97, 115409 (2018).

[17] K. M. Bastiaans, D. Cho, D. Chatzopoulos, M. Leeuwenhoek, C. Koks, and M. P. Allan, Imaging doubled shot noise in a Josephson scanning tunneling microscope, Phys. Rev. B 100, 104506 (2019).

[18] Y. Blanter and M. Büttiker, Shot noise in mesoscopic conductors, Phys. Rep. 336, 1 (2000).

[19] R. J. Schoelkopf, P. J. Burke, A. A. Kozhevnikov, D. E. Prober, and M. J. Rooks, Frequency Dependence of Shot Noise in a Diffusive Mesoscopic Conductor, Phys. Rev. Lett. 78, 3370 (1997).

[20] H. E. van den Brom and J. M. van Ruitenbeek, Quantum Suppression of Shot Noise in Atom-Size Metallic Contacts, Phys. Rev. Lett. 82, 1526 (1999).

[21] R. Cron, M. F. Goffman, D. Esteve, and C. Urbina, Multiple-Charge-Quanta Shot Noise in Superconducting Atomic Contacts, Phys. Rev. Lett. 86, 4104 (2001). 
[22] N. Agraït, A. Levy Yeyati, and J. M. van Ruitenbeek, Quantum properties of atomic-sized conductors, Phys. Rep. 377, 81 (2003).

[23] D. Djukic and J.M. van Ruitenbeek, Shot noise measurements on a single molecule, Nano Lett. 6, 789 (2006).

[24] N. L. Schneider, G. Schull, and R. Berndt, Optical Probe of Quantum Shot-Noise Reduction at a Single-Atom Contact, Phys. Rev. Lett. 105, 026601 (2010).

[25] S. Tewari, C. Sabater, M. Kumar, S. Stahl, B. Crama, and J. M. van Ruitenbeek, Fast and accurate shot noise measurements on atomic-size junctions in the $\mathrm{MHz}$ regime, Rev. Sci. Instrum. 88, 093903 (2017).

[26] K. M. Bastiaans, D. Cho, T. Benschop, I. Battisti, Y. Huang, M. S. Golden, Q. Dong, Y. Jin, J. Zaanen, and M. P. Allan, Charge trapping and super-Poissonian noise centres in a cuprate superconductor, Nat. Phys. 14, 1183 (2018).

[27] A. A. Odintsov, Effect of dissipation on the characteristics of small-area tunnel junctions: Application of the polaron model, Zh. Eksp. Teor. Fiz. 94, 312 (1988) [JETP 67, 1265 (1988)], http://jetp.ac.ru/cgi-bin/dn/e_067_06_1265.pdf.

[28] P. Delsing, K. K. Likharev, L. S. Kuzmin, and T. Claeson, Effect of High-Frequency Electrodynamic Environment on the Single-Electron Tunneling in Ultrasmall Junctions, Phys. Rev. Lett. 63, 1180 (1989).

[29] M. H. Devoret, D. Esteve, H. Grabert, G.-L. Ingold, H. Pothier, and C. Urbina, Effect of the Electromagnetic Environment on the Coulomb Blockade in Ultrasmall Tunnel Junctions, Phys. Rev. Lett. 64, 1824 (1990).

[30] H. Grabert, Single charge tunneling: A brief introduction, Z. Phys. B 85, 319 (1991).

[31] G.-L. Ingold and H. Grabert, Finite-temperature currentvoltage characteristics of ultrasmall tunnel junctions, Europhys. Lett. 14, 371 (1991).

[32] H. Grabert and G.-L. Ingold, Effect of the electromagnetic environment on single charge tunneling, in Computations for the Nano-Scale (Springer Netherlands, Dordrecht, 1993), p. 245.

[33] R. Cron, E. Vecino, M. H. Devoret, D. Esteve, P. Joyez, A. L. Yeyati, A. Martin-Rodero, and C. Urbina, Dynamical Coulomb blockade in quantum point contacts, in Proceedings, 36th Rencontres de Moriond, Electronic Correlations: From Meso- to Nano-Physics, edited by T. Martin, G. Montambaux, and J. Tran Thanh Van (EDP Sciences, Les Ulis, 2001).

[34] C. Altimiras, U. Gennser, A. Cavanna, D. Mailly, and F. Pierre, Experimental Test of the Dynamical Coulomb Blockade Theory for Short Coherent Conductors, Phys. Rev. Lett. 99, 256805 (2007).

[35] F. D. Parmentier, A. Anthore, S. Jezouin, H. le Sueur, U. Gennser, A. Cavanna, D. Mailly, and F. Pierre, Strong backaction of a linear circuit on a single electronic quantum channel, Nat. Phys. 7, 935 (2011).

[36] C. R. Ast, B. Jäck, J. Senkpiel, M. Eltschka, M. Etzkorn, J. Ankerhold, and K. Kern, Sensing the quantum limit in scanning tunneling spectroscopy, Nat. Commun. 7, 13009 (2016).

[37] D. V. Averin, Y. V. Nazarov, and A. A. Odintsov, Incoherent tunneling of the Cooper pairs and magnetic flux quanta in ultrasmall Josephson junctions, Physica (Amsterdam) (165166)B, 945 (1990).

[38] G.-L. Ingold and Y. V. Nazarov, Charge tunneling rates in ultrasmall junctions, in Single Charge Tunneling: Coulomb Blockade Phenomena in Nanostructures, edited by H. Grabert and M. H. Devoret (Springer US, Boston, MA, 1992), p. 21.

[39] J. Senkpiel, S. Dambach, M. Etzkorn, R. Drost, C. Padurariu, B. Kubala, W. Belzig, A. Levy Yeyati, J. C. Cuevas, J. Ankerhold, C. R. Ast, and K. Kern, Single channel Josephson effect in a high transmission atomic contact, arXiv:1810.10609.

[40] A. Levy Yeyati, A. Martin-Rodero, D. Esteve, and C. Urbina, Direct Link Between Coulomb Blockade and Shot Noise in a Quantum-Coherent Structure, Phys. Rev. Lett. 87, 046802 (2001).

[41] In the mesosocopic community the number of transport channels and their transmission is referred to as the PIN code in analogy to the "Personal Identification Number" used in financial transactions.

[42] A. Burtzlaff, N. L. Schneider, A. Weismann, and R. Berndt, Shot noise from single atom contacts in a scanning tunneling microscope, Surf. Sci. 643, 10 (2016).

[43] F. Massee, Q. Dong, A. Cavanna, Y. Jin, and M. Aprili, Atomic scale shot-noise using cryogenic $\mathrm{MHz}$ circuitry, Rev. Sci. Instrum. 89, 093708 (2018).

[44] F. Massee, Y. K. Huang, M. S. Golden, and M. Aprili, Noisy defects in the high- $T_{c}$ superconductor $\mathrm{Bi}_{2} \mathrm{Sr}_{2} \mathrm{CaCu}_{2} \mathrm{O}_{8+x}$, Nat. Commun. 10, 544 (2019).

[45] K. M. Bastiaans, T. Benschop, D. Chatzopoulos, D. Cho, Q. Dong, Y. Jin, and M. P. Allan, Amplifier for scanning tunneling microscopy at $\mathrm{MHz}$ frequencies, Rev. Sci. Instrum. 89, 093709 (2018).

[46] T. Aref, A. Averin, S. van Dijken, A. Ferring, M. Koberidze, V. F. Maisi, H. Q. Nguyend, R. M. Nieminen, J. P. Pekola, and L. D. Yao, Characterization of aluminum oxide tunnel barriers by combining transport measurements and transmission electron microscopy imaging, J. Appl. Phys. 116, 073702 (2014).

[47] L. J. Zeng, S. Nik, T. Greibe, P. Krantz, C. M. Wilson, P. Delsing, and E. Olsson, Direct observation of the thickness distribution of ultra thin $\mathrm{AlO}_{x}$ barriers in $\mathrm{Al} / \mathrm{AlO}_{x} / \mathrm{Al}$ Josephson junctions, J. Phys. D 48, 395308 (2015).

[48] M. Assig, M. Etzkorn, A. Enders, W. Stiepany, C. R. Ast, and K. Kern, A $10 \mathrm{mK}$ scanning tunneling microscope operating in ultra high vacuum and high magnetic fields, Rev. Sci. Instrum. 84, 033903 (2013).

[49] G.-L. Ingold, H. Grabert, and U. Eberhardt, Cooper-pair current through ultrasmall Josephson junctions, Phys. Rev. B 50, 395 (1994).

[50] See Supplemental Material at http://link.aps.org/ supplemental/10.1103/PhysRevLett.124.156803 for details on the sample preparation, the modeling of the $P(E)$ function, the transmission dependent DCB model, and the DFT-NEGF formalism, which includes Refs. [51-58].

[51] A. O. Caldeira and A. J. Leggett, Quantum tunnelling in a dissipative system, Ann. Phys. (N.Y.) 149, 374 (1983).

[52] Y. V. Nazarov, Influence of the electrodynamic environment on electron tunneling at finite traversal time, Phys. Rev. B 43, 6220 (1991). 
[53] J. P. Pekola, V. F. Maisi, S. Kafanov, N. Chekurov, A. Kemppinen, Y. A. Pashkin, O.-P. Saira, M. Möttönen, and J. S. Tsai, Environment-Assisted Tunneling as an Origin of the Dynes Density of States, Phys. Rev. Lett. 105, 026803 (2010).

[54] B. Jäck, M. Eltschka, M. Assig, M. Etzkorn, C. R. Ast, and K. Kern, Critical Josephson current in the dynamical Coulomb blockade regime, Phys. Rev. B 93, 020504(R) (2016).

[55] B. Jäck, M. Eltschka, M. Assig, A. Hardock, M. Etzkorn, C. R. Ast, and K. Kern, A nanoscale gigahertz source realized with Josephson scanning tunneling microscopy, Appl. Phys. Lett. 106, 013109 (2015).

[56] J. Tersoff and D. R. Hamann, Theory of the scanning tunneling microscope, Phys. Rev. B 31, 805 (1985).

[57] J. Bardeen, Tunnelling from a Many-Particle Point of View, Phys. Rev. Lett. 6, 57 (1961).

[58] S. Jezouin, M. Albert, F. D. Parmentier, A. Anthore, U. Gennser, A. Cavanna, I. Safi, and F. Pierre, TomonagaLuttinger physics in electronic quantum circuits, Nat. Commun. 4, 1802 (2013).

[59] D. S. Golubev and A. D. Zaikin, Coulomb Interaction and Quantum Transport Through a Coherent Scatterer, Phys. Rev. Lett. 86, 4887 (2001).

[60] F. Pauly, J. K. Viljas, U. Huniar, M. Häfner, S. Wohlthat, M. Bürkle, J. C. Cuevas, and G. Schön, Cluster-based densityfunctional approach to quantum transport through molecular and atomic contacts, New J. Phys. 10, 125019 (2008).

[61] E. Scheer, P. Joyez, D. Esteve, C. Urbina, and M. H. Devoret, Conduction Channel Transmissions of AtomicSize Aluminum Contacts, Phys. Rev. Lett. 78, 3535 (1997).
[62] J. C. Cuevas, A. Levy Yeyati, A. Martín-Rodero, G. R. Bollinger, C. Untiedt, and N. Agraï, Evolution of Conducting Channels in Metallic Atomic Contacts Under Elastic Deformation, Phys. Rev. Lett. 81, 2990 (1998).

[63] E. Scheer, N. Agraït, J. C. Cuevas, A. Levy Yeyati, B. Ludoph, A. Martín-Rodero, G. R. Bollinger, J. M. van Ruitenbeek, and C. Urbina, The signature of chemical valence in the electrical conduction through a single-atom contact, Nature (London) 394, 154 (1998).

[64] If the total transmission deviates slightly from the sum of the first three channel transmissions this is caused by the transmission of the nonvanishing higher channels and rounding errors.

[65] J. C. Cuevas, A. Levy Yeyati, and A. Martín-Rodero, Microscopic Origin of Conducting Channels in Metallic Atomic-Size Contacts, Phys. Rev. Lett. 80, 1066 (1998).

[66] M. Bürkle, J. K. Viljas, D. Vonlanthen, A. Mishchenko, G. Schön, M. Mayor, T. Wandlowski, and F. Pauly, Conduction mechanisms in biphenyl dithiol single-molecule junctions, Phys. Rev. B 85, 075417 (2012).

[67] M. Bürkle, J. K. Viljas, T. J. Hellmuth, E. Scheer, F. Weigend, G. Schön, and F. Pauly, Influence of vibrations on electron transport through nanoscale contacts, Phys. Status Solidi B 250, 2468 (2013).

[68] The dimer configuration provides a relatively unstable junction (in particular at high conductances), such that after careful analysis unfortunately only one reliable data point could be extracted. 